then this equality holds true for every integrable function $f(x)$ such that $\{f(x)\}^{2}$ is integrable.

GöTTINGEN,

July 17, 1908.

\title{
ON THE LOGICAL BASIS OF GRASSMANN'S EXTENSIVE ALGEBRA.
}

BY MR. A, R. SCHWEITZER.

\section{$\S 1$.}

Is studying the algebra of Grassmann fundamentally, we must carefully distinguish between the Ausdehnungslehre proper and the Ausdehnungslehre in a broad sense. Grassmann himself makes no rigid separation of the two viewpoints ; generally, however, the former is found in the edition of 1844 and the latter is in the edition of 1862 and in various memoirs.* Briefly, we may say that the Ausdehnungslehre proper for $n$ dimensions $(n=1,2,3, \ldots)$ is a development of $n$-dimensional euclidean geometry by means of the outer product of $n+1$ points, which fundamentally is reducible to sameness of sense of two $(n+1)$-hedra. It consists of descriptive axioms and certain axioms which relate exclusively to $n$-spatial congruence. On the basis of these axioms and their consequences, we arrive at the broader conception of the Ausdehnungslehre by means of suitable abstraction, the introduction of parameters, "formalization," etc. $\dagger$

\section{$\S 2$.}

If we take three dimensions, the fundamental properties of the Ausdehnungslehre are as follows. Concretely expressed, the basal relation is sameness of sense of two tetrahedra (identical or not) which is implied by what Grassmann has called "Gleichbezeichnung." $\$$ This relation is fundamentally nonmetrical, and solely in terms of it we may construct a system of postulates for three-dimensional descriptive geometry which is

\footnotetext{
* Cf. the Collected Works of Grassmann. For references to the Ausdehnungslehre and related subjects we may refer to Macfarlane's admirable bibliography, Dublin, 1904.

† For instance, see Crelle, vol. 49, p. 123 ; Math. Annalen, vol. 12, p. 376 ; Ausdehnungslehre, 1862, $z \&$ 151-215; Study, Wiener Berichte, vol. 91, p. 111. $\ddagger$ Coll. Works, I, p. 303, 304.
} 
sufficient for projective geometry. That two tetrahedra are "gleichartig" $*$ implies that they have the same or opposite senses, $i$. e., they are cospatial. Also four points $\alpha, \beta, \gamma, \delta$ form a tetrahedron if and only if the tetrad $\alpha \beta \gamma \delta$ is in the relation of sameness of sense to itself; abstractly this is expressed by $\alpha \beta \gamma \delta \mathrm{K} \alpha \beta \gamma \delta$ or $\alpha \beta \gamma \delta \mathrm{K}$. We note that the element point and relation $\mathrm{K}$ are undefined symbols which are effective under the conditions specified in the postulates which involve them. If then $\alpha \beta \gamma \delta \mathrm{K}$, we have $\beta \alpha \gamma \delta \mathrm{K}, \alpha \beta \gamma \delta \mathrm{K} \beta \gamma \alpha \delta, \alpha \beta \gamma \delta \mathrm{K} \gamma \delta \alpha \beta$, but $\alpha \beta \gamma \delta \overline{\mathrm{K}} \beta \alpha \gamma \delta$; $\dagger i$. e., $\alpha \beta \gamma \delta$ and $\beta \gamma \alpha \delta$ have the same sense, etc. The axiom of dimensionality is that $\alpha \beta \gamma \delta \mathrm{K}, \alpha^{\prime} \beta^{\prime} \gamma^{\prime} \delta^{\prime} \mathrm{K}$ imply $\alpha \beta \gamma \delta \mathrm{K} \alpha^{\prime} \beta^{\prime} \gamma^{\prime} \delta^{\prime}$ or $\alpha \beta \gamma \delta \mathrm{K} \beta^{\prime} \alpha^{\prime} \gamma^{\prime} \delta$. This axiom is a characteristic of sameness of sense as distinguished from similarity of direction (gleichläufig). $\$$ Axioms of geometric connection are : if $\alpha \beta \gamma \delta$ is a tetrahedron, and $\xi$ is any point, then $\xi \beta \gamma \delta$ or $\alpha \xi \gamma \delta$ or $\alpha \beta \xi \delta$ or $\alpha \beta \gamma \xi$ is a tetrahedron; if $\alpha \beta \gamma \delta$ and $\xi \beta \gamma \delta$ are two tetrahedra and $\xi \neq \alpha$, then $\alpha \xi \gamma \delta$ or $\alpha \beta \xi \delta$ or $\alpha \beta \gamma \xi$ is a tetrahedron; if $\alpha \beta \gamma \delta$ is a tetrahedron and $\xi$ lies on the faces $\alpha \beta \gamma$ and $\alpha \beta \delta$, then $\xi$ is collinear with $\alpha \beta$ (i.e., $\alpha \beta \xi \delta \overline{\mathrm{K}}$ for any $\delta)$. The following existential axioms are needed: there exists the tetrahedron $\alpha_{0} \beta_{0} \gamma_{0} \delta_{0}$; if $\alpha \beta \gamma \delta$ is a tetrahedron, there exists a point $\xi$ such that $\alpha$ is in the interior of the tetrahedron $\beta \gamma \delta \xi$; if $\xi$ is in the interior of $\alpha \beta \gamma \delta$, there exists an $\eta$ such that $\eta$ is on the plane $\xi \gamma \delta$ and betweeen $\alpha \beta$. il These axioms have a broader logical significance: the relation of sameness of sense is linearly transitive and symmetrical; also the tetrahedra $\xi \beta \gamma \delta$ and $\alpha \beta \gamma \delta$ have the same sense if $\xi$ is collinear with $\alpha \beta$ and $\xi \beta \gamma \delta$ and $\alpha \xi \gamma \delta$ have the same sense, or if $\xi$ is on the plane $\alpha \beta \gamma$ and $\xi \beta \gamma \delta, \alpha \xi \gamma \delta, \alpha \beta \xi \delta$ have the same sense, or if the tetrahedra $\xi \beta \gamma \delta, \alpha \xi \gamma \delta, \alpha \beta \xi \delta, \alpha \beta \gamma \xi$ have the same sense. Finally, if we add to the above properties the property of Dedekind continuity, which can be easily formulated in terms of sameness of sense in several elegant ways, we have all the descriptive properties necessary.§

\footnotetext{
* Grassmann, Coll. Works, I, p. 163.

+ The rule over the $K$ is a symbol of negation.

$\ddagger$ Grassmann, Coll. Works, I, p. 49 , note 3 .

|| For the definition of betweenness, etc., cf. the definitions for the plane by the author, BuLleTIN, November, 1906.

\& For the precise abstract statement of these properties, proof of their sufficiency, as well as full formulation of the definitions of betweenness, complanarity, etc., see a paper by the author offered to the Transactions for publication.
} 


\section{$\S 3$.}

To the terms of the preceding relation of sameness of sense we give the character of magnitudes by expressing $\alpha \beta \gamma \delta \mathrm{K} \alpha^{\prime} \beta^{\prime} \gamma^{\prime} \delta^{\prime}$ by $\alpha \beta \gamma \delta \equiv \alpha \beta \gamma \delta / \alpha^{\prime} \beta^{\prime} \gamma^{\prime} \delta^{\prime} \cdot \alpha^{\prime} \beta^{\prime} \gamma^{\prime} \delta^{\prime}$ and postulating that the symbols $\left(\alpha \beta \gamma \delta / \alpha^{\prime} \beta^{\prime} \gamma^{\prime} \delta^{\prime}\right)$ are a complete set of real positive numbers $(\mathrm{k}){ }^{*}$ The statement $\alpha \beta \gamma \delta \overline{\mathrm{K}}$ is expressed by $\alpha \beta \gamma \delta \equiv 0$. $\alpha^{\prime} \beta^{\prime} \gamma^{\prime} \delta^{\prime}$, i. e., $\alpha \beta \gamma \delta \equiv 0 ; \alpha \beta \gamma \delta \mathrm{K}$ is expressed by $\alpha \beta \gamma \delta \equiv \mathrm{I}$. $\alpha \beta \gamma \delta$ or $\alpha \beta \gamma \delta \neq 0$. We can now verify certain portions of $\S \S 96,97$ of Grassmann's Ausdehnungslehre of 1844 ; for example, if $\alpha \beta \gamma \delta \neq 0$, then $\alpha \beta \gamma \delta \equiv \mathrm{k} \alpha_{0} \beta_{0} \gamma_{0} \delta_{0}$ where $\mathrm{k}$ is a real number; $\dagger$ if $\alpha_{1} \beta_{1} \gamma_{1} \delta_{1} \equiv \mathrm{k}_{1} \alpha_{0} \beta_{0} \gamma_{0} \delta_{0}$ and $\alpha_{2} \beta_{2} \gamma_{2} \delta_{2} \equiv \mathrm{k}_{2} \alpha_{0} \beta_{0} \gamma_{0} \delta_{0}$ then for the sum we have $\alpha_{1} \beta_{1} \gamma_{1} \delta_{1}+\alpha_{2} \beta_{2} \gamma_{2} \delta_{2} \equiv\left(\mathrm{k}_{1}+\mathrm{k}_{2}\right) \alpha_{0} \beta_{0} \gamma_{0} \delta_{0}$, etc. Other properties of spatial congruence are that if $\alpha \beta \gamma \delta \neq 0$, $\alpha \beta \gamma \delta^{\prime} \neq 0$ and $\delta \delta^{\prime}$ is parallel to $\alpha \beta \gamma$, then $\alpha \beta \gamma \delta \equiv \mathrm{I} \cdot \alpha \beta \gamma \delta^{\prime}$; and that the sum $\xi \beta \gamma \delta+\alpha \xi \gamma \delta+\alpha \beta \xi \delta+\alpha \beta \gamma \xi \equiv \mathrm{I} \cdot \alpha \beta \gamma \delta$. We are now able to prove the grassmannian numerical derivation of points, uniqueness of parallel line, derivation of vectors, etc.\$ The further procedure consists in admitting the point with weight zero and developing projective geometry. Grassmann clearly indicates the character of this development in the Ausdehnungslehre of 1844 . In this connection one is reminded of a hiatus in Grassmann's calculus, namely, the absence of an absolute theory of angles. Such a theory is certainly desirable from a pedagogical as well as theoretical viewpoint; and the prospect for it on the basis of the preceding properties is very promising. However, from the above, we are already in a position to verify in a fundamental, logical manner that "Grassmann's Ausdehnungslehre is a shape into which projective geometry may be thrown.” §

* Cf. Grassmann, Coll. Works, I, p. 138.

$\dagger$ If $\mathrm{k}>0$ then $a \beta \gamma \delta$ and $a_{0} \beta_{0} \gamma_{0} \delta_{0}$ are "gleichbezeichnet"; if $\mathrm{k} \neq 0, a \beta \gamma \delta$ and $a_{0} \beta_{0} \gamma_{0} \delta_{0}$ are "gleichartig."

†Cf. Grassmann, Coll. Works, I, \& 110 ; Peano, Calcolo Geometrico.

\& Lasker, Proc: London Math. Society, vol. 28, pp. 217, 218. 